\title{
Multicomponent crystal forms of a biologically active hydrazone with some dicarboxylic acids: Salts or cocrystals?
}

\author{
Liliana Mazur, ${ }^{a *}$ Ilona Materek, ${ }^{a}$ Andrew D. Bond ${ }^{b}$ and William Jones ${ }^{b}$
}

a Faculty of Chemistry, Maria Curie-Skłodowska University, Maria Curie-Skłodowska Square 2, 20-031 Lublin, Poland

${ }^{b}$ Department of Chemistry, University of Cambridge, Lensfield Road, Cambridge CB2 1EW, United Kingdom

* Corresponding author: Liliana Mazur (l.mazur@poczta.umcs.lublin.pl)

\section{Cocrystal screening}

Table 1S. Physicochemical characteristics $\left(T_{\mathrm{m}}\right.$ - melting point, $T_{\text {fus }}$ - temperature of fusion) of the studied adducts.

\begin{tabular}{|c|c|c|c|c|c|}
\hline \multirow{2}{*}{ Form } & \multirow{2}{*}{$\begin{array}{c}T_{\mathrm{m}} \text { (acid) } \\
/{ }^{\circ} \mathrm{C}\end{array}$} & \multirow{2}{*}{$\begin{array}{l}T_{\mathrm{m}} \text { (adduct) } \\
/{ }^{\circ} \mathrm{C} \text { (HSM) }\end{array}$} & \multicolumn{2}{|c|}{$T_{\text {fus }}$ (adduct) $/{ }^{\circ} \mathrm{C}$} & \multirow{2}{*}{$\begin{array}{c}\text { IR } \\
\left(v_{\max }, \mathrm{cm}^{-1}\right)\end{array}$} \\
\hline & & & (onset) & (peak) & \\
\hline $\mathbf{B Z H} \cdot \mathrm{H}_{2} \mathbf{O}$ & - & $128-130$ & 121 & 125 & See Ref. 42. \\
\hline 1 & $132-135$ & $100-119$ & 108 & 113 & $\begin{array}{l}3249,3224,3047,2970,2937,1725,1694,1617,1603,1578, \\
1544,1489,1470,1450,1422,1375,1351,1259,1183,1153, \\
1092,1034,1023,1001,957,933,921\end{array}$ \\
\hline 2 & $184-186$ & $118-139$ & 137 & 140 & $\begin{array}{l}\text { 3205, 3116, 3082, 3065, 3032, } 2970,2943,1718,1688,1636, \\
1602,1556,1494,1467,1417,1352,1318,1264,1252,1182, \\
1141,1096,1076,1029,938,917\end{array}$ \\
\hline 3 & $95-98$ & $103-111$ & 101 & 105 & $\begin{array}{l}3268,3084,2933,2658,2593,1716,1642,1602,1581,1557, \\
1474,1458,1437,1421,1386,1363,1326,1308,1290,1249, \\
1220,1184,1170,1147,1103,1080,1054,1042,1030,1004, \\
941,926\end{array}$ \\
\hline $4 a$ & & $115-133$ & 124 & $\begin{array}{l}128 \\
132\end{array}$ & $\begin{array}{l}3261,3065,2799,2720,2440,1687,1637,1602,1591,1578, \\
1557,1498,1470,1448,1436,1362,1346,1331,1312,1287, \\
1266,1208,1150,1126,1105,1080,1008,967,943,922\end{array}$ \\
\hline 4b & $200-202$ & $112-138$ & 132 & 136 & $\begin{array}{l}3299,3064,2918,2848,2567,1719,1698,1681,1644,1605, \\
1591,1580,1551,1493,1475,1493,1386,1370,1343,1309, \\
1257,1214,1192,1147,1127,1097,1077,1026,1014,1001, \\
941,919,908\end{array}$ \\
\hline $4 \mathrm{a} \cdot \mathrm{CH}_{3} \mathrm{OH}$ & & $\begin{array}{c}60-90 \\
119-133\end{array}$ & 124 & $\begin{array}{l}127 \\
131\end{array}$ & $\begin{array}{l}\text { 3326, 3114, 3082, 3034, 2942, 2720, 2502, 1717, 1689, 1642, } \\
1601,1580,1556,1494,1467,1446,1418,1374,1320,1252, \\
1182,1141,1143,1096,1076,1028,1000,938,917\end{array}$ \\
\hline
\end{tabular}




\section{X-ray crystallography}

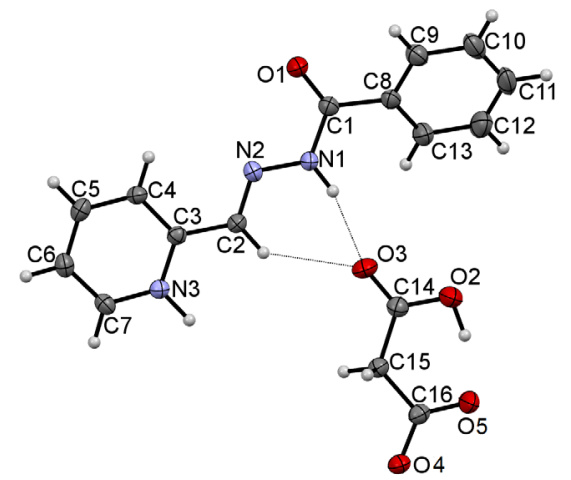

1

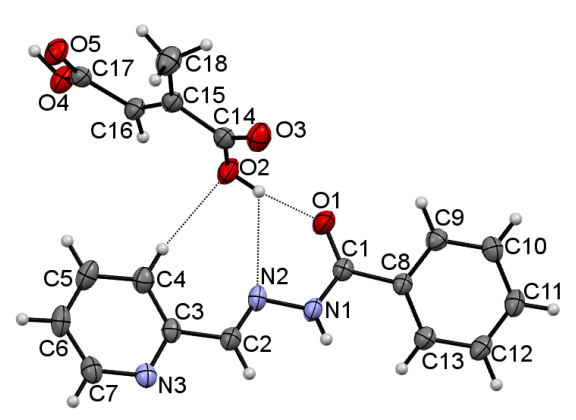

$4 a$

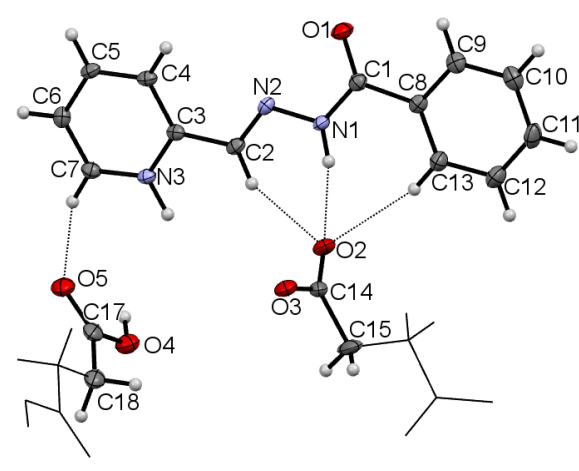

2

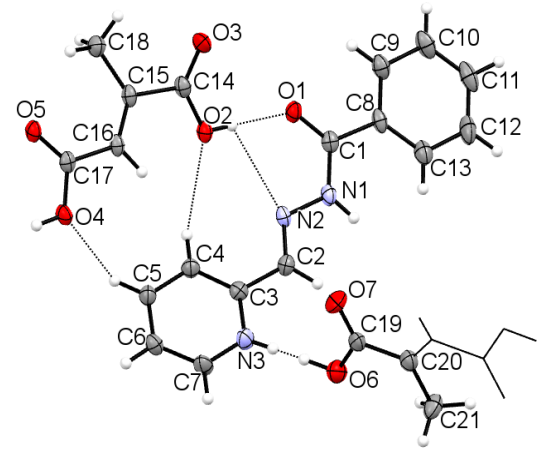

4b

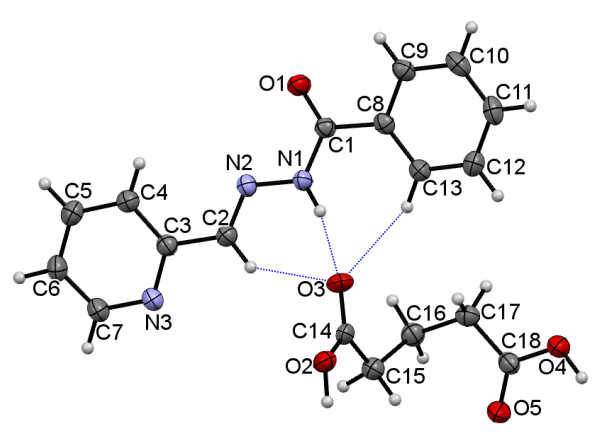

3

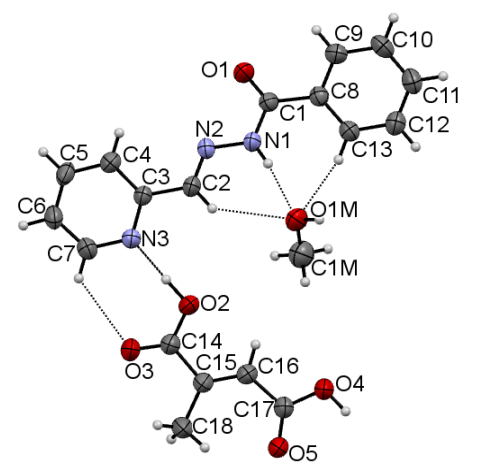

$4 a \cdot \mathrm{CH}_{3} \mathrm{OH}$

Figure 1S. Labelling of atoms and estimation of their thermal motion parameters as ADPs (50\% probability level) for studied forms. Dashed lines indicate hydrogen bonds. 
Table 2S. Selected geometric parameters ( $d$ - bond distance, $\theta$ - bond angle, $\tau$ - torsion angle).

\begin{tabular}{|c|c|c|c|c|c|c|c|}
\hline$d / \AA$ & $\begin{array}{c}\mathbf{B Z H} \cdot \mathbf{H}_{\mathbf{2}} \mathbf{O} \\
\text { CIZRAE02 }^{42}\end{array}$ & 1 & 2 & 3 & $4 a$ & $4 \mathrm{~b}$ & $4 \mathrm{a} \cdot \mathrm{CH}_{3} \mathrm{OH}$ \\
\hline C1-01 & $1.229(1)$ & $1.223(2)$ & $1.224(3)$ & $1.235(2)$ & $1.229(3)$ & $1.224(3)$ & $1.220(2)$ \\
\hline $\mathrm{C} 1-\mathrm{N} 1$ & $1.362(1)$ & $1.378(2)$ & $1.372(3)$ & $1.354(2)$ & $1.345(3)$ & $1.356(3)$ & $1.358(2)$ \\
\hline $\mathrm{N} 1-\mathrm{N} 2$ & $1.368(1)$ & $1.360(2)$ & $1.368(3)$ & $1.375(2)$ & $1.379(3)$ & $1.375(3)$ & $1.375(2)$ \\
\hline $\mathrm{N} 2-\mathrm{C} 2$ & $1.283(1)$ & $1.283(2)$ & $1.281(3)$ & $1.278(2)$ & $1.281(3)$ & $1.281(3)$ & $1.271(2)$ \\
\hline C3-N3 & $1.344(1)$ & $1.348(2)$ & $1.352(3)$ & $1.343(2)$ & $1.351(3)$ & $1.345(3)$ & $1.347(2)$ \\
\hline N3-C7 & $1.339(1)$ & $1.340(2)$ & $1.345(3)$ & $1.343(2)$ & $1.345(3)$ & $1.338(3)$ & $1.338(2)$ \\
\hline C14-02 & & $1.311(2)$ & $1.289(3)$ & $1.322(2)$ & $1.316(3)$ & $1.324(3)$ & $1.308(2)$ \\
\hline C14-03 & & $1.216(2)$ & $1.236(3)$ & $1.216(2)$ & $1.218(3)$ & $1.206(3)$ & $1.205(2)$ \\
\hline $\mathrm{C} 16 / \mathrm{C} 17-04$ & & $1.269(2)$ & $1.329(3)$ & $1.325(2)$ & $1.331(3)$ & $1.318(3)$ & $1.314(2)$ \\
\hline C16/C17-05 & & $1.243(2)$ & $1.223(3)$ & $1.206(2)$ & $1.203(3)$ & $1.230(3)$ & $1.217(2)$ \\
\hline C19-06 & & & & & & $1.300(3)$ & \\
\hline C19-07 & & & & & & $1.217(3)$ & \\
\hline \multicolumn{8}{|l|}{$\theta /^{\circ}$} \\
\hline C3-N3-C7 & 118.1(1) & $121.6(2)$ & $122.5(2)$ & $117.4(1)$ & $117.6(2)$ & $118.9(2)$ & $118.0(2)$ \\
\hline 01-C1-N1 & $123.0(1)$ & $122.1(2)$ & $122.4(2)$ & $122.2(1)$ & $122.3(2)$ & $122.1(2)$ & $121.9(2)$ \\
\hline $\mathrm{N} 1-\mathrm{N} 2-\mathrm{C} 2$ & $115.3(1)$ & $117.1(1)$ & $115.4(2)$ & $116.2(1)$ & $115.3(2)$ & $116.6(2)$ & $117.0(2)$ \\
\hline \multicolumn{8}{|l|}{$\tau /{ }^{\circ}$} \\
\hline 01-C1-N1-N2 & $-1.7(1)$ & $2.1(2)$ & $1.5(4)$ & $-5.8(2)$ & $2.1(4)$ & $2.7(4)$ & $-0.3(3)$ \\
\hline $\mathrm{C} 1-\mathrm{N} 1-\mathrm{N} 2-\mathrm{C} 2$ & $-176.9(1)$ & $-177.6(2)$ & $177.9(2)$ & $-177.7(1)$ & $179.0(2)$ & $-169.8(2)$ & $177.9(2)$ \\
\hline $\mathrm{N} 1-\mathrm{N} 2-\mathrm{C} 2-\mathrm{C} 3$ & $178.9(1)$ & $179.5(1)$ & $179.2(2)$ & $178.0(1)$ & $-179.1(2)$ & $-177.2(2)$ & $178.6(2)$ \\
\hline $\mathrm{N} 2-\mathrm{C} 2-\mathrm{C} 3-\mathrm{N} 3$ & $-175.1(1)$ & 174.1(1) & $178.5(2)$ & 168.1(1) & $178.6(2)$ & $-171.8(2)$ & $168.2(2)$ \\
\hline 01-C1-C8-C13 & $-158.2(1)$ & $-153.0(2)$ & $-179.4(3)$ & $-166.6(2)$ & $-170.4(2)$ & $175.4(2)$ & $-165.6(2)$ \\
\hline
\end{tabular}

Table 3S. Geometries of hydrogen bonds and selected short contacts for studied structures.

\begin{tabular}{|c|c|c|c|c|c|c|}
\hline Form & Interaction & $d_{\mathrm{D} \ldots \mathrm{H}} / \AA$ & $d_{\mathrm{H} \ldots \mathrm{A}} / \AA$ & $d d_{\mathrm{D} \ldots \mathrm{A}} / \AA$ & $<\mathrm{D}-\mathrm{H} \ldots \mathrm{A} /{ }^{\circ}$ & Symmetry code \\
\hline \multirow[t]{10}{*}{1} & $\mathrm{~N} 1-\mathrm{H} 1 \mathrm{n} \cdots 03$ & $0.91(2)$ & $1.99(2)$ & $2.878(2)$ & 165(2) & $x, y, z$ \\
\hline & $\mathrm{C} 2-\mathrm{H} 2 \cdots \mathrm{O} 3$ & 0.95 & 2.44 & $3.235(2)$ & 141 & \\
\hline & N3-H3n $\cdots 04$ & $1.04(3)$ & $1.53(3)$ & $2.564(2)$ & $179(3)$ & \\
\hline & N3-H3n $\cdots 05$ & $1.04(3)$ & $2.61(3)$ & $3.275(2)$ & $122(2)$ & $-x,-y+1,-z+1$ \\
\hline & $\mathrm{C} 7-\mathrm{H} 7 \cdots \mathrm{O} 5$ & 0.95 & 2.59 & $3.240(2)$ & 125 & \\
\hline & $\mathrm{O} 2-\mathrm{H} 2 \mathrm{o} \cdots 04$ & $0.98(3)$ & $1.58(3)$ & $2.530(2)$ & $163(3)$ & $x, y, z$ \\
\hline & $\mathrm{C} 11-\mathrm{H} 11 \cdots 05$ & 0.95 & 2.65 & $3.500(2)$ & 149 & $-x,-y+2,-z+1$ \\
\hline & $\mathrm{C} 5-\mathrm{H} 5 \cdots \mathrm{O} 1$ & 0.95 & 2.45 & $3.197(2)$ & 135 & $-x+2,-y+1,-z$ \\
\hline & $\mathrm{C} 15-\mathrm{H} 15 \mathrm{a} \cdots 04$ & 0.99 & 2.67 & $3.448(2)$ & 136 & $-x,-y+1,-z+1$ \\
\hline & $\mathrm{C} 15-\mathrm{H} 15 \mathrm{~b} \cdots 04$ & 0.99 & 2.62 & $3.498(2)$ & 149 & $-x-1,-y+1,-z+1$ \\
\hline \multirow[t]{6}{*}{2} & $\mathrm{~N} 1-\mathrm{H} 1 \mathrm{n} \cdots \mathrm{O} 3$ & $0.89(3)$ & $1.93(3)$ & $2.801(3)$ & $166(2)$ & \\
\hline & $\mathrm{C} 2-\mathrm{H} 2 \cdots 03$ & 0.93 & 2.44 & $3.176(3)$ & 137 & $x, y, z$ \\
\hline & $\mathrm{C} 13-\mathrm{H} 13 \cdots \mathrm{O} 3$ & 0.93 & 2.45 & $3.331(4)$ & 157 & \\
\hline & $\mathrm{N} 3-\mathrm{H} 3 \mathrm{n} \cdots \mathrm{O} 2$ & $0.99(3)$ & $1.61(3)$ & $2.599(3)$ & $176(2)$ & $x, y-1, z$ \\
\hline & $\mathrm{O} 4-\mathrm{H} 4 \mathrm{O} \cdots 02$ & $0.87(4)$ & $1.73(4)$ & $2.594(3)$ & $177(2)$ & $x, y-2, z$ \\
\hline & $\mathrm{C} 5-\mathrm{H} 5 \cdots \mathrm{O} 1$ & 0.93 & 2.58 & $3.012(4)$ & 109 & $\mathrm{x}+1 / 2, \mathrm{y}-1 / 2,-\mathrm{z}+3 / 2$ \\
\hline
\end{tabular}




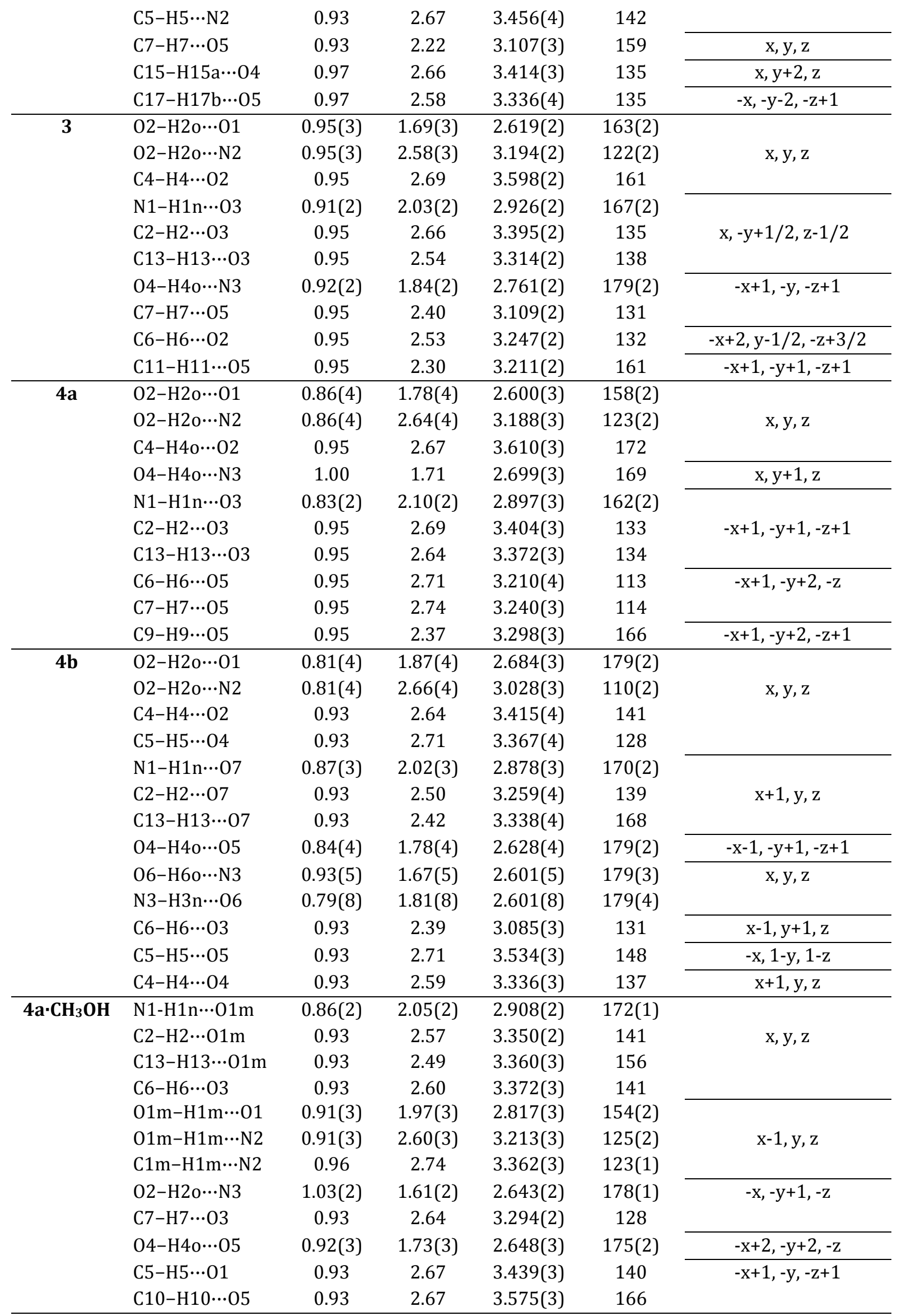




\section{Powder X-ray diffraction}

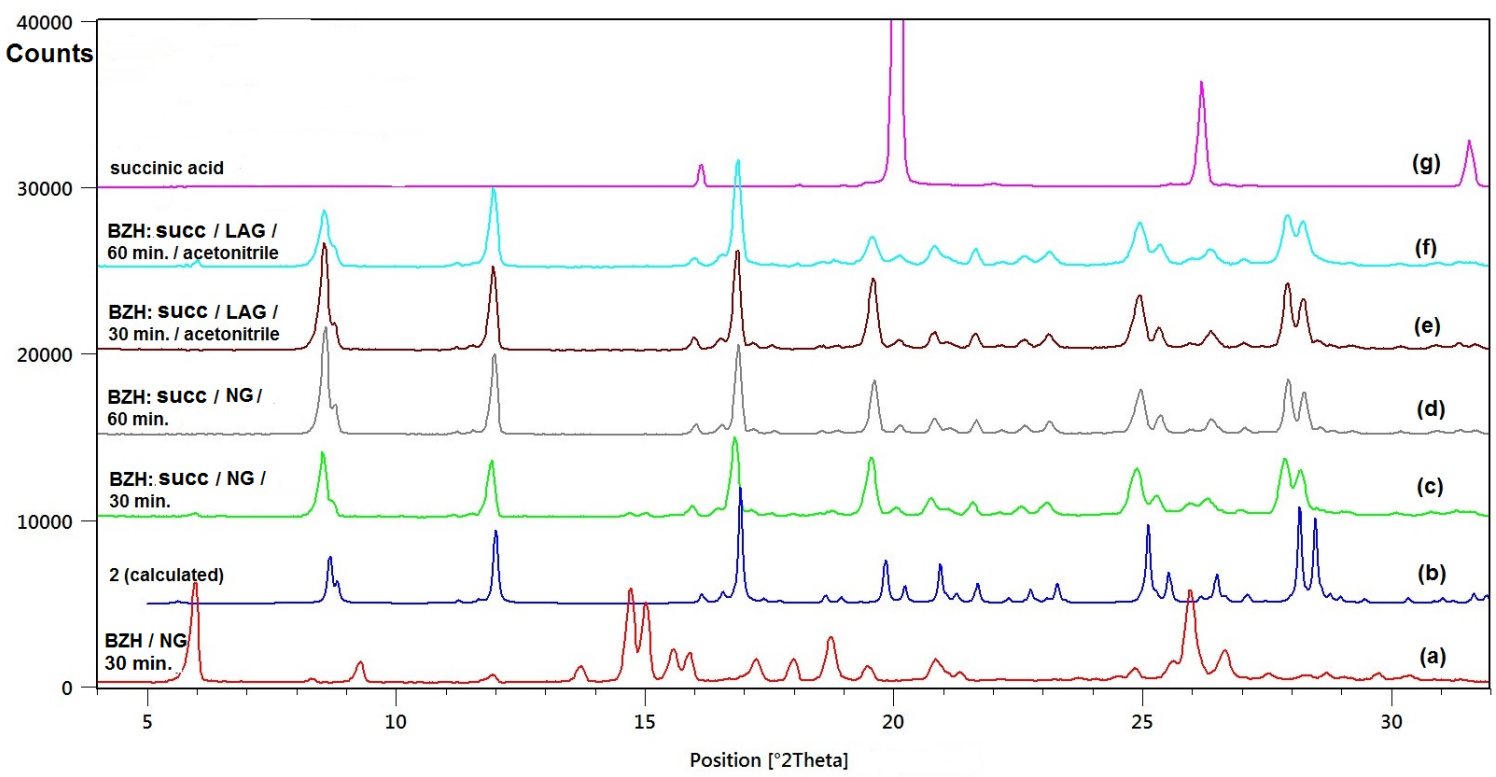

Figure 2S. PXRD patterns of: (a) pure BZH· $\mathbf{H}_{2} \mathbf{O}$ after NG; (b) calculated BZH-succ (2); (c), (d) BZH-succ prepared by NG after 30 and 60 minutes of milling; (e), (f) BZH-succ prepared by LAG using acetonitrile after 30 and 60 minutes of milling, respectively; (g) pure succinic acid.

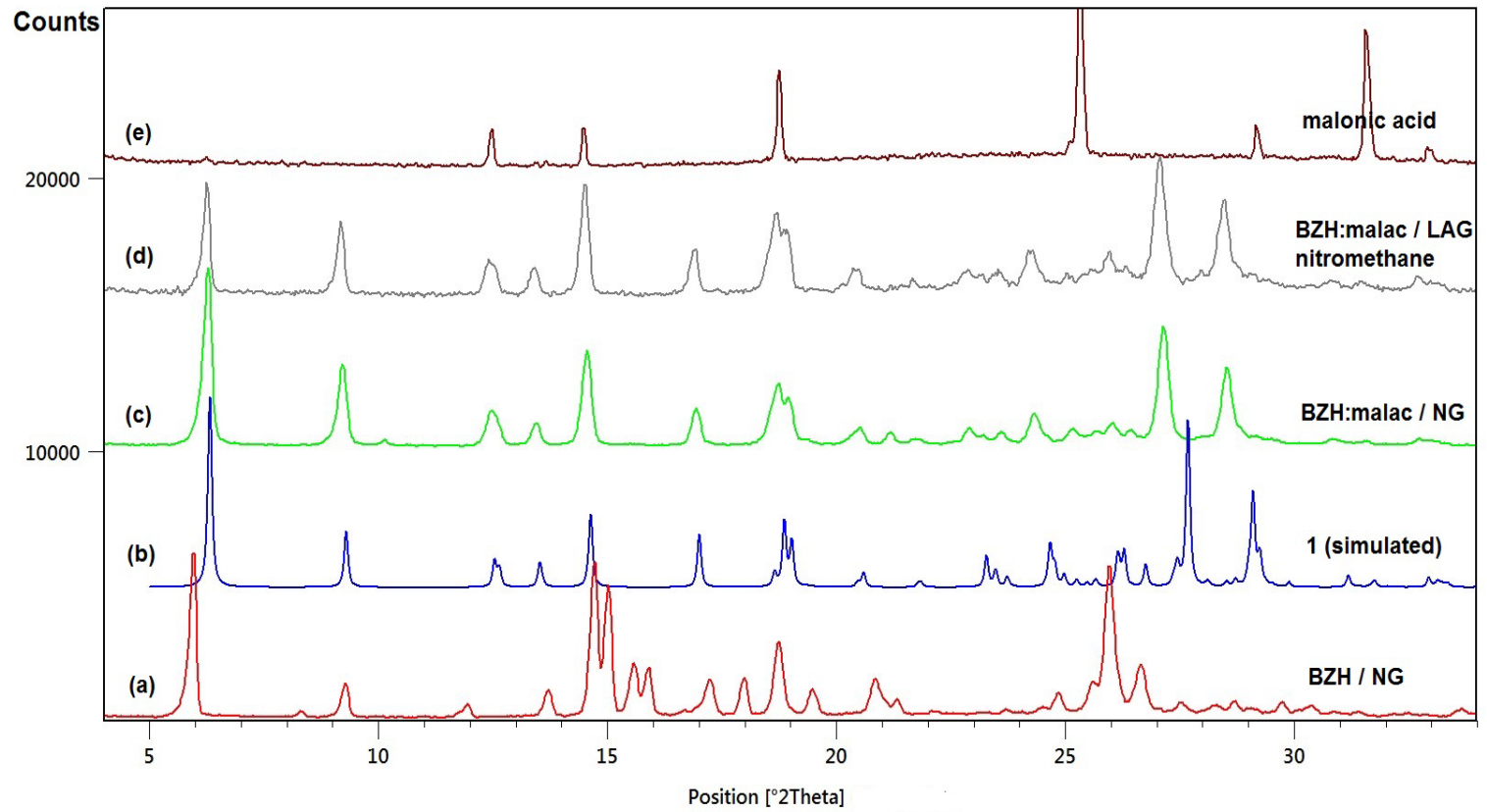

Figure 3S. PXRD patterns of: $(a)$ pure $\mathbf{B Z H} \cdot \mathbf{H}_{2} \mathbf{O}$ after neat grinding; (b) calculated BZH-malac (1); (c) BZH-malac prepared by NG; (d) BZH-malac prepared by LAG using nitromethane; (e) pure malonic acid. 


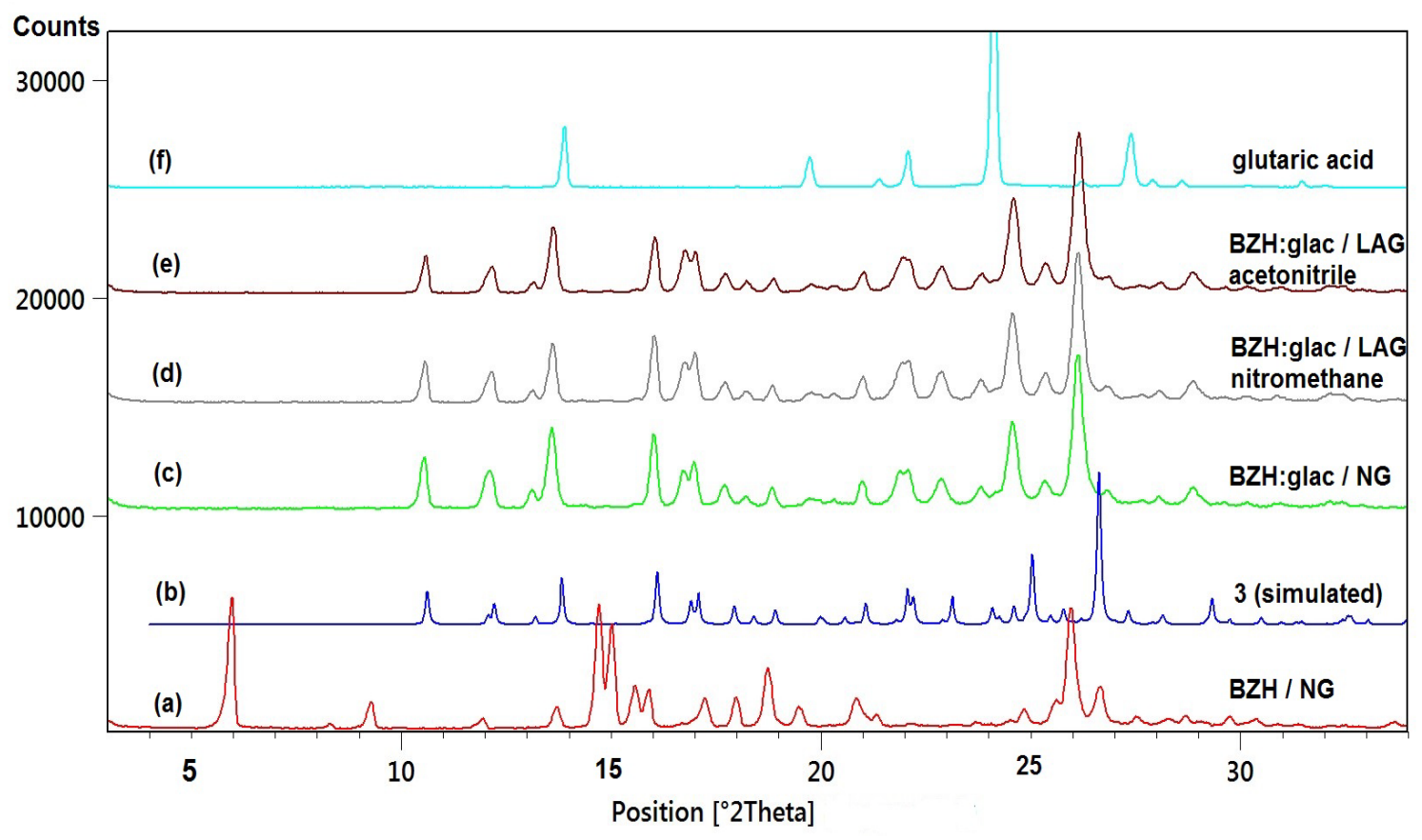

Figure 4S. PXRD patterns of: (a) pure BZH·H $\mathbf{H}_{2} \mathbf{O}$ after NG; (b) calculated BZH-glac (3); (c) BZHglac prepared by NG; (d), (e) BZH-glac prepared by LAG using nitromethane or acetonitrile; (f) pure glutaric acid. All PXRD patterns were recorded at room temperature.

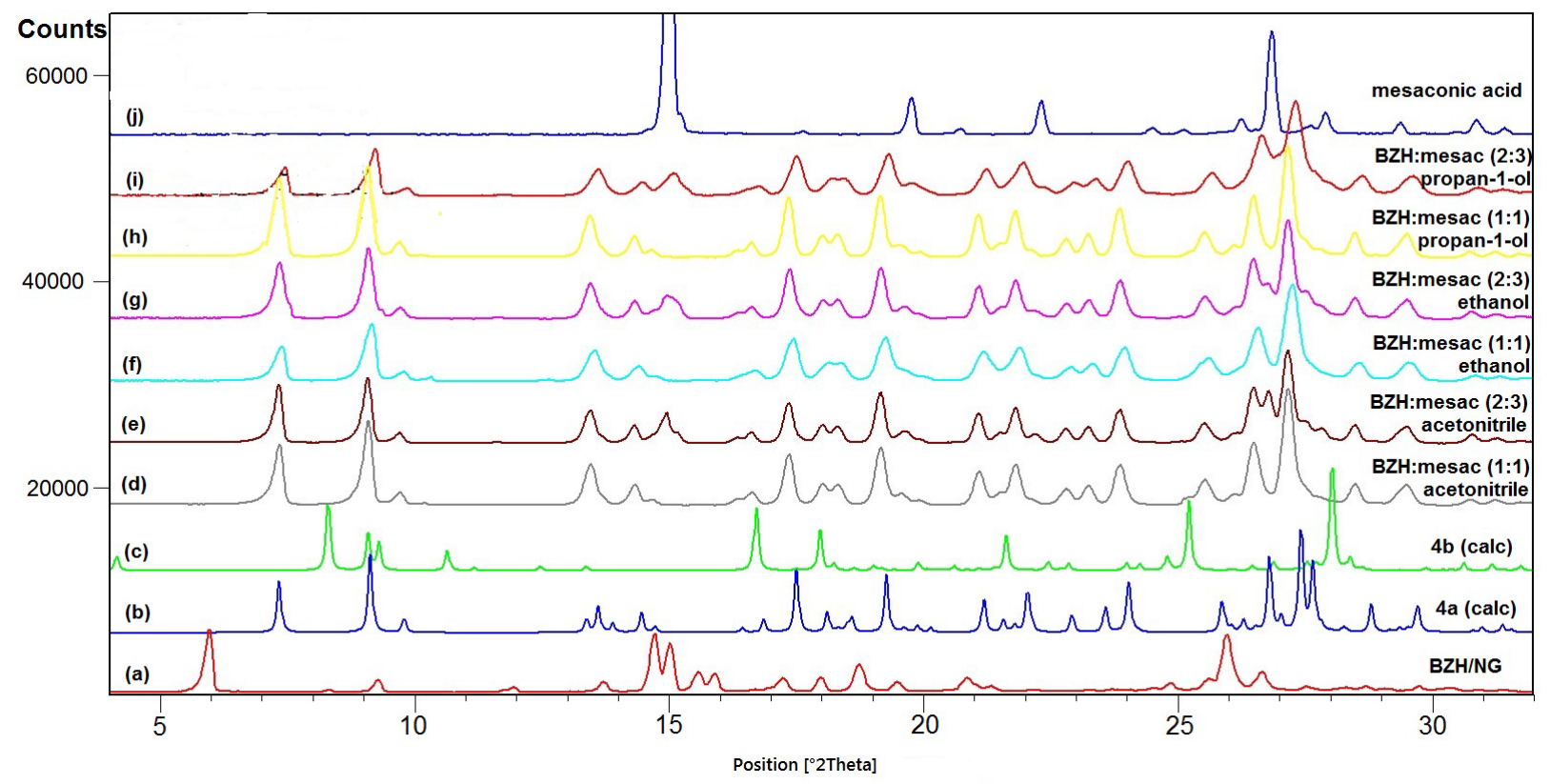

Figure 5S. PXRD patterns of: (a) pure BZH· $\mathbf{H}_{2} \mathbf{O}$ after NG; (b) calculated BZH-mesac (4a); (c) calculated BZH-mesac (4b); (d), (f), (h) BZH-mesac (1:1) and (e), (g), (i) BZH-mesac (2:3) after LAG using acetonitrile; ethanol or propan-1-ol; (j) pure mesaconic acid. 


\section{Synthon occurrences (CSD searches)}

Table 4S. Modes of binding of 2-pyridinecarboxaldehyde $N^{1}$-acylhydrazones - carboxylic acid solids (CSD survey).

\begin{tabular}{|c|c|c|c|c|c|c|}
\hline Entry & $\begin{array}{l}\text { CSD } \\
\text { reference } \\
\text { code }\end{array}$ & Hydrate & $\begin{array}{l}\text { Hydrogen-bond } \\
\text { donor group }\end{array}$ & $\begin{array}{l}\text { Hydrogen-bond } \\
\text { acceptor group }\end{array}$ & Synthon & Ref \\
\hline \multicolumn{7}{|c|}{ N'-(pyridin-2-ylmethylene)isonicotinohydrazide / 2-Pyridylcarboxaldehyde isonicotinoyl hydrazone } \\
\hline 1. & LATCIU & $\sqrt{ }$ & carboxyl & 4-pyridyl & B & 4 \\
\hline 2. & LATCOA & $\sqrt{ }$ & carboxyl & 4-pyridyl & B & 4 \\
\hline 3. & LATCUG & $\sqrt{ }$ & carboxyl & 4-pyridyl & $\mathrm{B}$ & 4 \\
\hline 4. & LATDAN & $\sqrt{ }$ & carboxyl & 4-pyridyl & B & 4 \\
\hline 5. & LATDER & $\sqrt{ }$ & carboxyl & 4-pyridyl & $\mathrm{B}$ & 4 \\
\hline 6. & LATDIV & $\sqrt{ }$ & carboxyl & 4-pyridyl & $\mathrm{A}$ & 4 \\
\hline 7. & LATDOB & $\sqrt{ }$ & carboxyl & 4-pyridyl & B & 4 \\
\hline 8. & LATDUH & $\sqrt{ }$ & carboxyl & 4-pyridyl & A & 4 \\
\hline \multicolumn{7}{|c|}{ N'-(pyridin-3-ylmethylene)pyridine-2-carbohydrazide } \\
\hline 9. & KECKEL & & $\begin{array}{l}\text { carboxyl, } \\
\text { amide }\end{array}$ & $\begin{array}{c}\text { 3-pyridyl, amide, } \\
\text { carboxyl }\end{array}$ & \begin{tabular}{|l|} 
B, D \\
C \\
\end{tabular} & 51 \\
\hline 10. & KECKIP & & $\begin{array}{l}\text { carboxyl, } \\
\text { amide }\end{array}$ & $\begin{array}{l}\text { 3-pyridyl, amide, } \\
\text { carboxyl }\end{array}$ & $\begin{array}{l}\text { B, D } \\
\text { C }\end{array}$ & 51 \\
\hline 11. & KECLOW & & carboxyl & 3-pyridyl, amide & A, D & 51 \\
\hline 12. & KECMAJ & & carboxyl & 3-pyridyl, amide & A, D & 51 \\
\hline 13. & KECSUJ & & carboxyl & 3-pyridyl & $\mathrm{B}$ & 51 \\
\hline \multicolumn{7}{|c|}{ N'-(pyridin-3-ylmethylene)nicotinohydrazide } \\
\hline 14. & KECKOV & $\sqrt{ }$ & carboxyl & 3-pyridyl & A, B & 51 \\
\hline \multicolumn{7}{|c|}{ N'-(pyridin-4-ylmethylene)nicotinohydrazide } \\
\hline 15. & KECKUB & $\sqrt{ }$ & carboxyl & 3-pyridyl & $\mathrm{A}$ & 51 \\
\hline 16. & KECSOD & & carboxyl & 4-pyridyl, amide & A, D & 51 \\
\hline 17. & KECTAQ & $\sqrt{ }$ & carboxyl & 3-pyridyl, 4-pyridyl & A, B & 51 \\
\hline \multicolumn{7}{|c|}{ 4-Pyridylcarboxaldehyde isonicotinoylhydrazone } \\
\hline 18. & LATFET & $\sqrt{ }$ & carboxyl & 4-pyridyl & & 4 \\
\hline 19. & LATFIX & $\sqrt{ }$ & carboxyl & 4-pyridyl & A, B & 4 \\
\hline 20. & LATFOD & $\sqrt{ }$ & carboxyl & 4-pyridyl & A, B & 4 \\
\hline \multicolumn{7}{|c|}{ 4-(2-((2-hydroxyphenyl)methylidene)hydrazinecarbonyl)pyridin-1-ium } \\
\hline 21. & OKAVED & & $\begin{array}{l}\text { 4-pyridyl, } \\
\text { amide }\end{array}$ & $\begin{array}{c}\text { carboxyl, carboxylate } \\
\text { carboxylate }\end{array}$ & $\begin{array}{l}\mathrm{B}, \mathrm{B}^{\prime} \\
\mathrm{C} \\
\end{array}$ & 50 \\
\hline 22. & OKAVIH & & 4-pyridyl, amide & carboxylate & $A^{\prime}, C$ & 50 \\
\hline 23. & OKAVON & & 4-pyridyl, amide & carboxylate & $\mathrm{B}^{\prime}, \mathrm{C}$ & 50 \\
\hline 24. & OKAWAA & $\sqrt{ }$ & 4-pyridyl, amide & carboxylate & $\mathrm{B}^{\prime}, \mathrm{C}$ & 50 \\
\hline \multicolumn{7}{|c|}{ N'-(4-methylbenzylidene)isonicotinohydrazide } \\
\hline 25. & SAYQEQ & $\sqrt{ }$ & carboxyl & 4-pyridyl & A & S1 \\
\hline 26. & ZAQMIP & $\sqrt{ }$ & carboxyl & 4-pyridyl & A & S1 \\
\hline \multicolumn{7}{|c|}{ N'-(2-methoxybenzylidene)isonicotinohydrazide } \\
\hline 27. & LEFQUK & & carboxyl & 4-pyridyl & A & S2 \\
\hline \multicolumn{7}{|c|}{ N'-(4-methoxybenzylidene)isonicotinohydrazide } \\
\hline 28. & CEFZEU & & carboxyl & 4-pyridyl, amide & $\mathrm{B}, \mathrm{D}$ & S3 \\
\hline \multicolumn{7}{|c|}{ (E)-N'-(5-Chloro-3-methoxy-2-(4-methylphenylsulfonyloxy)benzylidene)isonicotinohydrazide } \\
\hline 29. & JIRWOX & & $\begin{array}{l}\text { carboxyl } \\
\text { amide }\end{array}$ & $\begin{array}{l}\text { amide, } \\
\text { carboxyl }\end{array}$ & $\begin{array}{l}\text { D } \\
\text { C } \\
\end{array}$ & S4 \\
\hline \multicolumn{7}{|c|}{ (E)-N'-(2-(4-Chloro-3-nitrophenylsulfonyloxy)-3-methoxybenzylidene)isonicotinohydrazide } \\
\hline 30. & IGABEY & & $\begin{array}{l}\text { carboxyl, } \\
\text { amide }\end{array}$ & $\begin{array}{l}\text { 4-pyridyl, amide, } \\
\text { carboxyl }\end{array}$ & $\begin{array}{l}\text { A, D } \\
\text { C }\end{array}$ & S5 \\
\hline \multicolumn{7}{|c|}{ (N'-furfurylidene)isonicotinoylhydrazide } \\
\hline 31. & NOSZAW & $\sqrt{ }$ & carboxyl & 4-pyridyl & A & S6 \\
\hline
\end{tabular}




\section{Thermal stability studies}

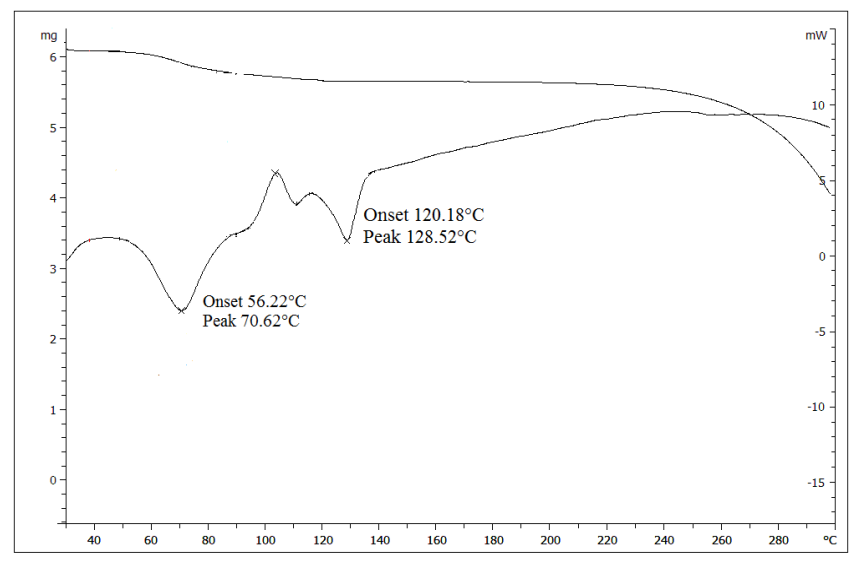

(a)

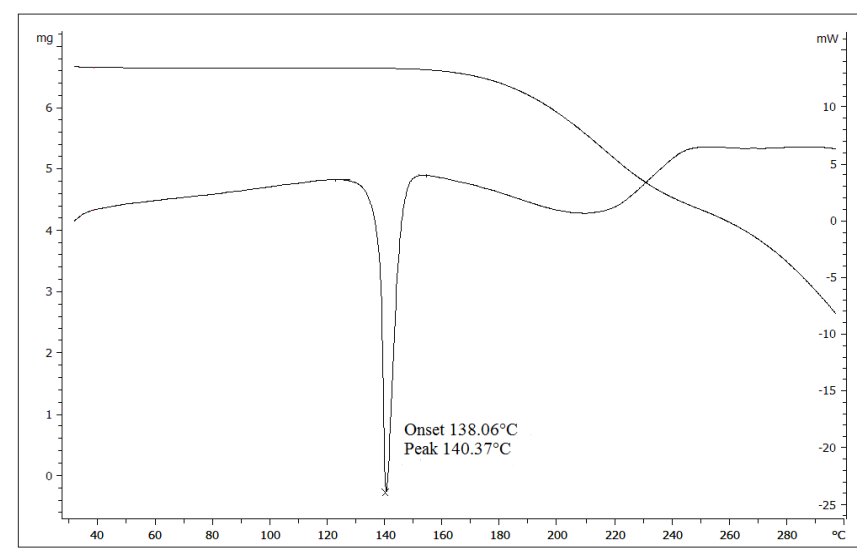

$(c)$

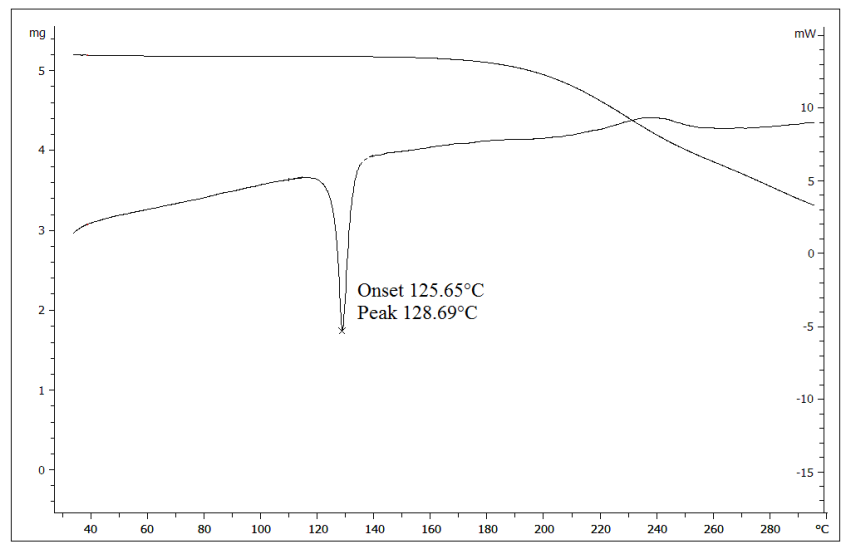

$(e)$

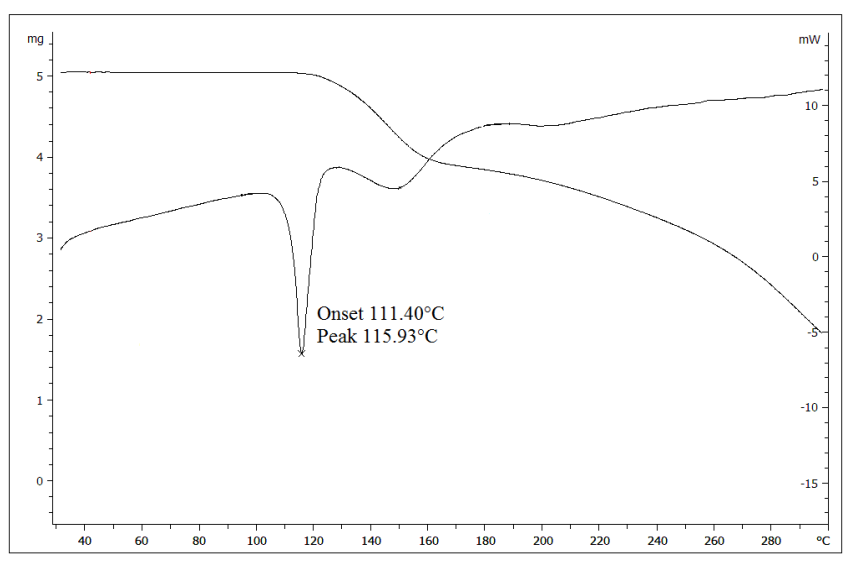

(b)

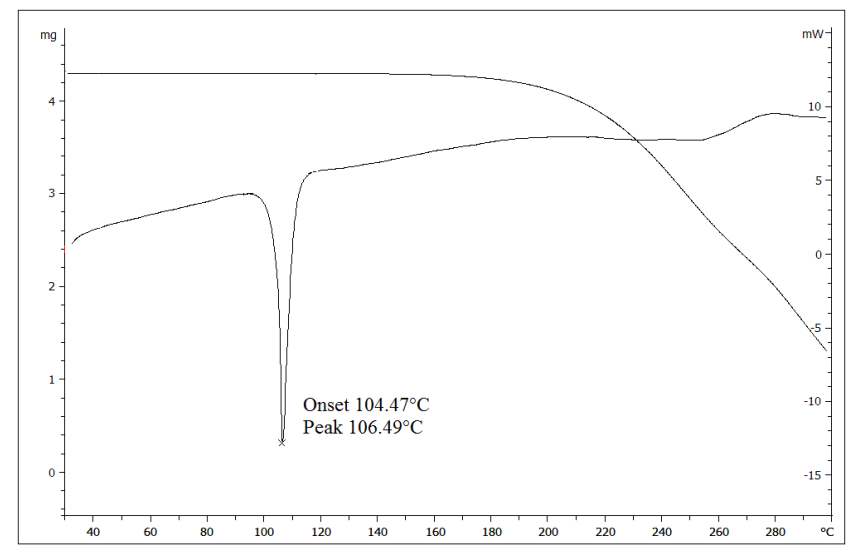

(d)

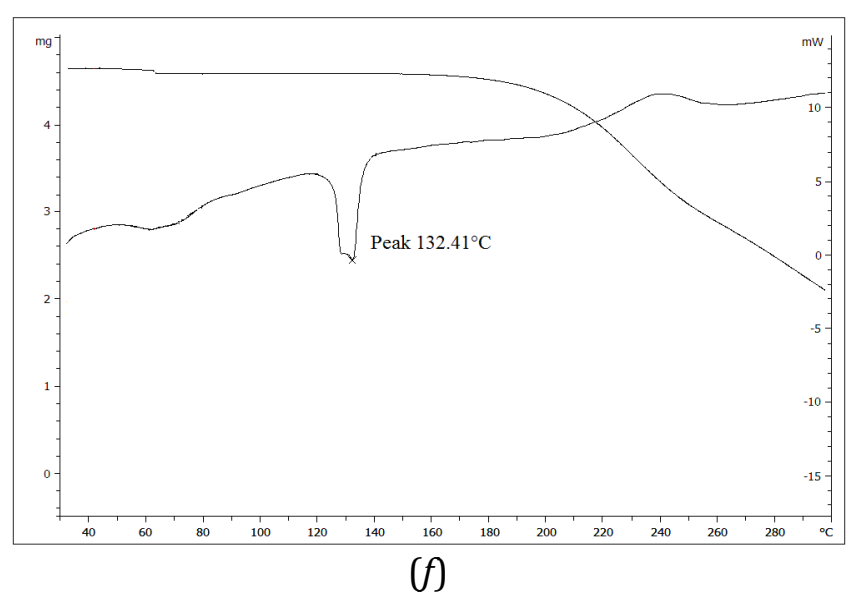

Figure 6S. TG/DSC plots for: $(a) \mathbf{B Z H} \cdot \mathbf{H}_{2} \mathbf{O},(b) \mathbf{1},(c)$ 2, $(d)$ 3, $(e)$ 4a, $(f)$ 4b recorded in the $\mathrm{N}_{2}$ atmosphere with a heating rate of $10^{\circ} \mathrm{C} \mathrm{min}^{-1}$. 


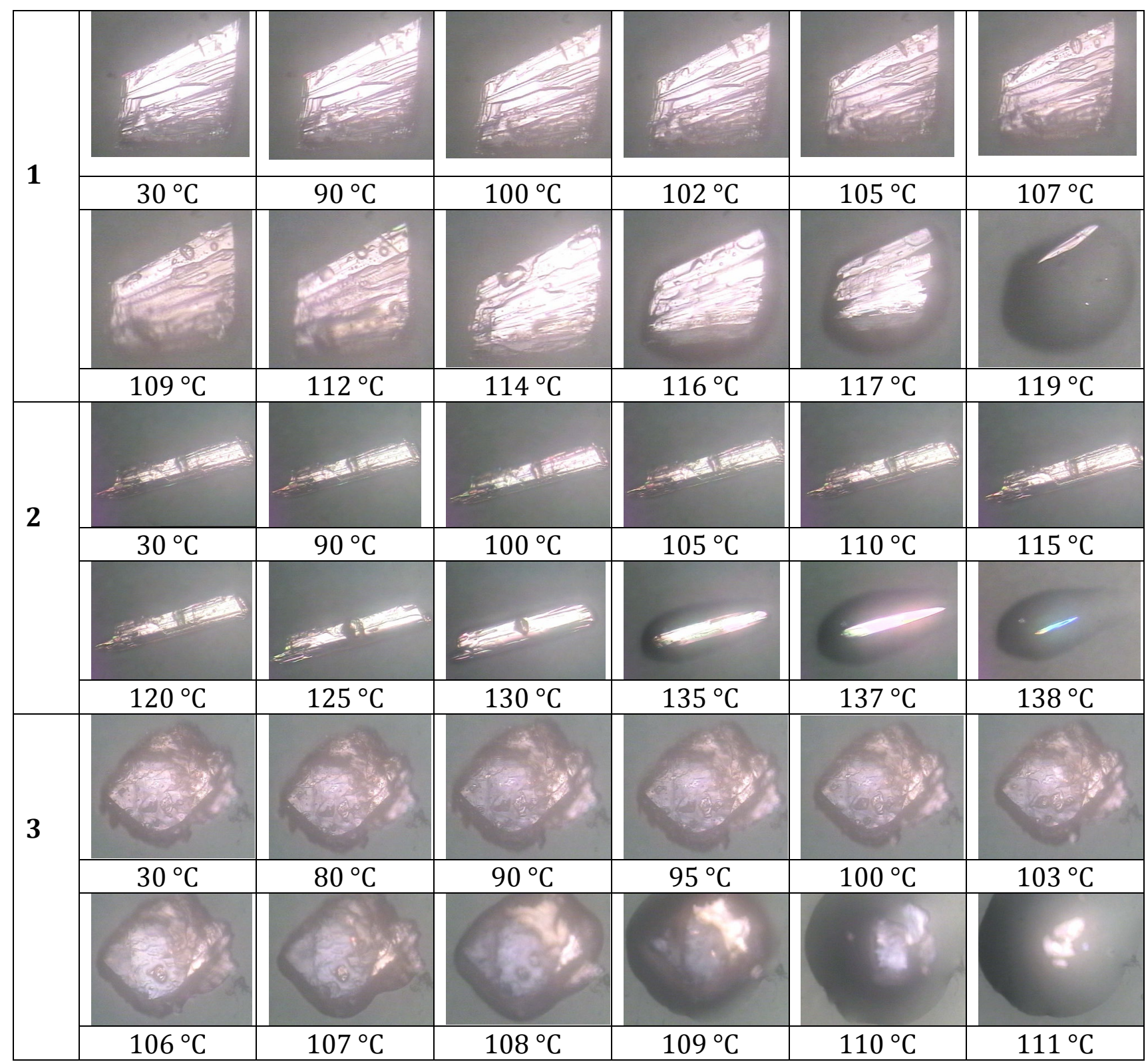

Figure 7S. Hot-stage micrographs of salts BZH-malac (1), BZH-succ (2) and cocrystal BZH-glac (3). The micrographs were recorded in the air with the heating rate of $2^{\circ} \mathrm{C} \min ^{-1}$.

\section{References}

S1. Lemmerer, A.; Bernstein, J.; Kahlenberg, V. Covalent assistance in supramolecular synthesis: in situ modification and masking of the hydrogen bonding functionality of the supramolecular reagent isoniazid in co-crystals. CrystEngComm 2011, 13, 5692-5708.

S2. Mohamed, S. K.; Farrukh, M. A.; Akkurt, M.; Albayati, M. R.; Abdelhamid, A. A. 2-(1,3-Dioxoisoindolin-2yl)acetic acid- $N$ '-[(E)-2-methoxybenzylidene]pyridine-4-carbohydrazide (1/1). Acta Crystallogr., Sect. E: Struct. Rep. Online 2012, 68, o2442. 
S3. Novakovic, S. B.; Bogdanovic, G. A.; Mohamed, S. K.; Albayati, M. R.; Hameed, A. S. 2-(1,3-Dioxoisoindolin-2-yl)acetic acid-N'-[(E)-4-methoxybenzylidene]pyridine-4-carbohydrazide (2/1). Acta Crystallogr., Sect. E: Struct. Rep. Online 2012, 68, o2897-o2898.

S4. Diao, Ch.-H.; Fan, Z.; Chen, X. (E)- $N^{\prime}$-[5-Chloro-3-methoxy-2-(4-methylphenylsulfonyloxy)benzylidene] isonicotinohydrazide acetic acid solvate: hydrogen-bonded network of alternating $R_{4}^{4}(42), R_{5}^{5}(33)$ and $R_{6}^{6}(40)$ rings. Acta Crystallogr., Sect. C: Cryst. Struct. Commun. 2007, 63, o717-o719.

S5. Zhen, X.-L.; Li, X.-L. (E)-N'-[2-(4-Chloro-3-nitro-phenyl-sulfon-yloxy)-3-methoxy-benzylidene]isonicotinohydrazide acetic acid tetra-solvate. Acta Crystallogr., Sect. E: Struct. Rep. Online 2008, 64, o2170.

S6. Chuev, I. I.; Nikonova, L. A.; Atovmyan, E. G.; Utenyshev, A. N.; Aldoshin, S. M. Molecular and Crystal Structure of the Products of Crystallization of ( $N^{\prime}$-Furfurylidene)isonicotinoylhydrazide from Aquaeous Solutions of hydrochloric and Acetic Acids. Kristallografiya 2001, 46, 444-447. 